\title{
3 bản ghi trong Thư viện ĐHKHXH\&NV - ĐHQGTPHCM
}

Trung tâm thông tin thư viện

Đại học Khoa học xã hội \& Nhân văn

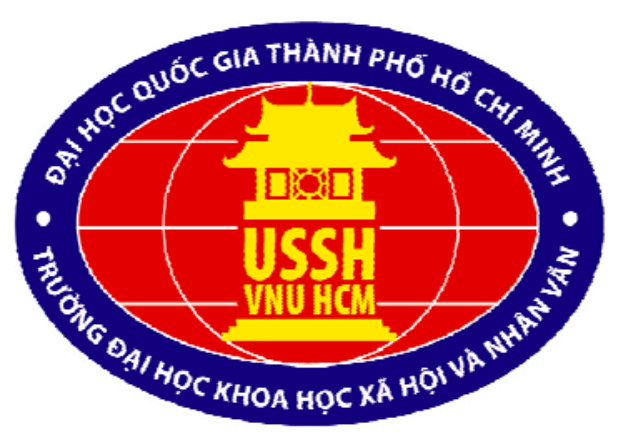

Đại học Quốc gia Tp. Hồ Chí Minh

Truy cập:

Ngày 1 tháng 3 năm 2021

Địa chỉ URL:

http://opac.hcmussh.edu.vn/cgi-

bin/gw_43_3/chameleon?host=localhost\%2B1111\%2BDEFAULT\&search=KEYWORD\&function=INITREQ \&SourceScreen=NEXTPAGE\&sessionid=2009102106183202220\&skin=default\&conf=.\%2Fchameleon.co nf\&Ing=vn\&itemu1=2000\&scant1=V\%C6\%B0\%C6\%A1ng\%20T\%E1\%BA\%A5t\%20\%C4\%90\%E1\%BA\%A1t, \&scanu1=1003\&u1=2000\&t1=@185635\&pos=1\&prevpos=0\&

Ba địa chỉ sách truy cập được qua hệ thống OPAC:

1. Văn minh làm giàu \& nguồn gốc của cải

http://opac.hcmussh.edu.vn/cgi-

bin/gw_43_3/chameleon?host=localhost\%2b1111\%2bDEFAULT\&search=KEYWORD\&function=INITREQ \&SourceScreen=INITREQ\&sessionid=2009102106183202220\&skin=default\&conf=.\%2fchameleon.conf \&Ing=vn\&itemu1=2000\&scant1=V\%c4\%83n\%20minh\%20l\%c3\%a0m\%20gi\%c3\%a0u\%20\%26\%20ngu\%e 1\%bb\%93n\%20g\%e1\%bb\%91c\%20c\%e1\%bb\%a7a\%20c\%e1\%ba\%a3i\%20\%2f\&scanu1=4\&u1=2000\&t1= @138721\&pos=1\&prevpos=1\&\#

Mã Q529 V115M 
Đánh số quyển (item): VVa29841; VVa29842; VVa29843; VVa29844

2. Kinh tế Việt Nam: Thăng trầm và đột phá

http://opac.hcmussh.edu.vn/cgi-

bin/gw_43_3/chameleon?host=localhost\%2b1111\%2bDEFAULT\&search=KEYWORD\&function=INITREQ \&SourceScreen=INITREQ\&sessionid=2009102106183202220\&skin=default\&conf $=. \% 2 \mathrm{fchameleon}$.conf \&lng=vn\&itemu1=2000\&scant1=Ph\%e1\%ba\%a1m,\%20Minh\%20Ch\%c3\%adnh.\&scanu1=1003\&u1=200 0\&t1=@205335\&pos=1\&prevpos=1\&

Mã Q9(1)0 K312T

Đánh số quyển: VVa41583; VVa41584; VVa41585; VVa41586

3. Bằng chứng cuộc sống: Suy nghĩ về phát triển bền vững Việt Nam

http://opac.hcmussh.edu.vn/cgi-

bin/gw_43_3/chameleon?host=localhost\%2b1111\%2bDEFAULT\&search=SCAN\&function=INITREQ\&So urceScreen=INITREQ\&sessionid=2009102106183202220\&skin=default\&conf $=. \% 2 \mathrm{fchameleon}$.conf\&Ing =vn\&itemu1=4\&scant1=V\%c6\%b0\%c6\%a1ng\%20T\%e1\%ba\%a5t\%20\%c4\%90\%e1\%ba\%a1t,\&scanu1=10 03\&u1=4\&t1=B\%e1\%ba\%b1ng\%20ch\%e1\%bb\%a9ng\%20cu\%e1\%bb\%99c\%20s\%e1\%bb\%91ng\%20\%20Suy\%20ngh\%c4\%a9\%20v\%e1\%bb\%81\%20ph\%c3\%a1t\%20tri\%e1\%bb\%83n\%20b\%e1\%bb\%81n\%20 v\%e1\%bb\%afng\%20Vi\%e1\%bb\%87t\%20Nam\%20\%2f\&pos=1\&prevpos=1\&beginsrch=1

Mã Q9(1) B116C

Đánh số quyển: VVa68604

Chi tiết thông tin thư viện như trong bản các bản pdf printout từ OPAC của thư viện USSH VNU HCM bên dưới.

Thông tin trích dẫn trong phần tài liệu tham khảo. 


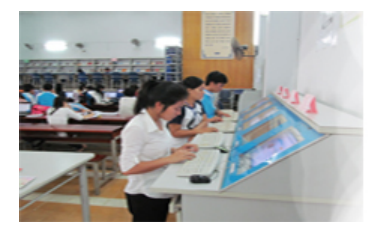

Trang chủ

\section{ĐAI HQE QUరC GIA THÀNH PHO HO CHÍ MINH ĐẠI HOC KHOA HOC XÄ HỌI VÀ NHÂN VĂN TRUNG TÂM THOONG TIN THUU VIỆN}

Đặt trước
Tìm ở các thư viện khác
Trợ giúp

Lưu phiên làm việc

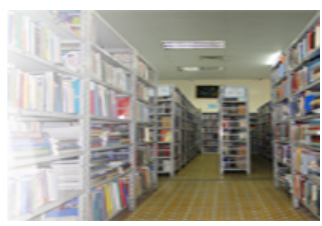

Đóng phiên làm việc

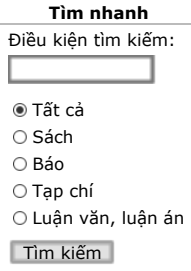

Dặt loc

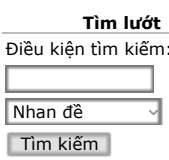

Database:

TITT-TV ĐHKHXH\&NV

ĐHQGTPHCM

Cóo tài liệu trong giỏ của
bann.

Lịch sử tìm kiếm

Số lượng truy cập

局

Recommended Reading

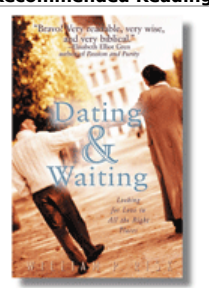

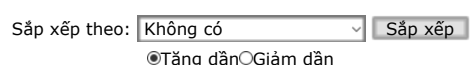

You searched TTTT-TV ĐHKHXH\&NV-ĐHOGTPHCM - Nhan đề: Landfall/

Records 1 through 3 of 3
Save to List Check $1-3$ Clear All

Q 1 Check shelf : 5

Card Vị trí : 1.Phòng đọc-DTH-Ref, 2.Phòng đọc-TD-Ref, 3.Phòng mượ-DTH-Ref, 4.Phòng mượn-TD-Ref

items(5) Author Vương, Quân Hoàng.

Marc Title Văn minh làm giàu \& nguồn gốc của cải

Giỏ hàng? Publication H. : Chính trị Quốc gia, 2007.

Material $497 \mathrm{tr} \cdot 24 \mathrm{~cm}$

2 Check shelf : 4

Card Vị trí : 1.Phòng đọc-DTH-Ref, 2.Phòng đọc-TD-Ref, 3.Phòng mượn-DTH-Ref, 4.Phòng mượn-TD-Ref

items(4) Author Phạm, Minh Chính.

Marc Title Kinh tế Việt Nam thăng trầm và đột phá /

Giỏ hàng? Publication H. : Chính trị Quốc gia, 2009.

Material 554 tr. ; $22 \mathrm{~cm}$.

3 Check shelf : 1

Card Vị trí : 1.Phòng đọc-DTH-Re

items(1) Author Bach, Ngọc Chiến.

Marc Bitle Bằng chứng cuộc sống - Suy nghĩ về phát triển bền vững Việt Nam/

Giỏ hàng? Publication Hà Nội : Chính trị Quỗc gia, 2015.

Material 255 tr.; $22 \mathrm{~cm}$.

Save to List Check 1-3 Clear All

Tìm kiếm theo từ khóa

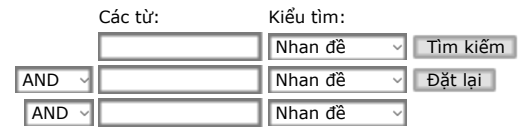

Cơ sở dữ liệu: TITT-TV ĐHKHXH\&NV-ĐHQGTPHCM

Set Search Filters

Các bước như sau:

1. Nhập thuật ngữ tìm vào ô "Các từ", chọn từ liên kết "AND", "NOT" để giới hạn hoặc "OR", "NEAR" để mở rộng kết quá tim.

2. Chon: nhan đề, tác giả, chủ đề...trong ô "Kiểu tìm".

3. Nhấn "Đặt lại" đế xóa rông dữ liệu các ô và nhập lại thuật

ngữ tìm kiếm.

4. Nhấn "Tìm kiếm".

http://opac.hcmussh.edu.vn/cgi-bin/gw_43_3/chameleon?host=localhost $\% 2 B 1111 \%$

2BDEFAULT\&search=KEYWORD\&function=INITREQ\&SourceScreen=NEXTPAGE\&sessionid=2 $009102106183202220 \&$ skin $=$ default\&conf $=. \% 2$ Fchameleon.conf\&lng $=$ vn\&itemu $1=2000 \&$ scant $1=\mathrm{V}$ $\% \mathrm{C} 6 \% \mathrm{~B} 0 \% \mathrm{C} 6 \% \mathrm{~A} 1 \mathrm{ng} \% 20 \mathrm{~T} \% \mathrm{E} 1 \% \mathrm{BA} \% \mathrm{~A} 5 \mathrm{t} \% 20 \% \mathrm{C} 4 \% 90 \% \mathrm{E} 1 \% \mathrm{BA} \%$

A1t,\&scanu $1=1003 \& u 1=2000 \& t 1=@ 185635 \&$ pos $=1 \&$ prevpos $=0 \&$ 


\section{Tài liệu tham khảo:}

[1] Vương Quân Hoàng. (2007). Văn minh làm giàu \& Nguồn gốc của cải. Nxb Chính trị Quốc gia, Hà Nội.

[2] Phạm Minh Chính, Vương Quân Hoàng. (2009). Kinh tế Việt Nam: Thăng trầm và Đột phá. Nxb Chính trị Quốc gia, Hà Nội.

[3] Bạch Ngọc Chiến, Vương Quân Hoàng. (2015). Bằng chứng cuộc sống: Suy nghĩ về phát triển bền vững Việt Nam. Nxb Chính trị Quốc gia, Hà Nội. 